\title{
Neurocysticercosis in Iran: An Unexpected Case and Literature Review
}

\section{Zeinab Kishani Farahani, Afshin Moradi, Amirsaiid Sedighi, Mohammadmehdi Soleimani}

Shahid Beheshti University of Medical Sience, Shohada Hospital, Tehran, Iran.

Email: afshinmo2002@yahoo.com

Received April $6^{\text {th }}, 2013$; revised May $8^{\text {th }}, 2013$; accepted June $4^{\text {th }}, 2013$

Copyright (C) 2013 Zeinab Kishani Farahani et al. This is an open access article distributed under the Creative Commons Attribution License, which permits unrestricted use, distribution, and reproduction in any medium, provided the original work is properly cited.

\begin{abstract}
Neurocysticercosis is a significant public health issue worldwide. Even though cysticercosis was once thought to have been eradicated in the United States, it is currently a growing public health problem in the United States, and immigrants from endemic areas are at the highest risk of acquiring this disease. In Iran, there was no report from this worrisome infection. The clinical presentation of neurocysticercosis is variable and vague. In this article, we will explain a case of neurocysticercosis encountered in the Shohada Hospital pathology department, and then we will review neurologic and radiologic symptoms, laboratory and pathologic findings, as well as medication for treatment, briefly.
\end{abstract}

Keywords: Neurocysticercosis; Epilepsy; Racemose; Scolex

\section{Introduction}

Neurocysticercosis is the most common helminth infection of the central nervous system, and accounts for about 50,000 deaths per year worldwide, and many times, this number of people are with active epilepsy $[1,2]$.

It is endemic in Mexico, Central and South America, Asia, India, Africa, and Indonesia [3].

With increased travel to disease-endemic areas and the migration of tapeworm carriers or people infected with the disease, neurocysticercosis is becoming increasingly prevalent even in industrialized countries [4].

\section{Case Presentation}

A 63 years old man presented to Shohada Hospital emergency department with headache and nausea for 2 months with unconsciousness worsened during 14 days. He had history of ischemic heart disease, diabetes mellitus and cardiac bypass surgery. His brain CT scans revealed multiple cystic deep parieto occipital lesions, which induced midline shifting and moderate hydrocephalus. The patient admitted in ICU and went under insertion of external ventricular drainage that improved the level of consciousness. The patient was candidate for stereotactic biopsy which diagnosed as neurocysticercosis in our pathology department. Our case radiologic and pathologic findings will be represented in forthcoming relevant sessions. Due to definite pathologic diagnosis, albendazole $400 \mathrm{mg}$ twice daily, dexamethasone $8 \mathrm{mg}$ 3 times daily administered for the patient, but showed no marked response unfortunately. He gradually deteriorated and was intubated for airway protection, placed on a mechanical ventilator but during next days the patient expired due to septicemia secondary to pneumonia.

\subsection{Life Cycle}

Neurocysticercosis is an infestation of the brain by Taenia solium, the tissue-invading larval form of the pork tapeworm. The larvae are introduced into the body by the accidental ingestion of fertile $T$. solium eggs in fecally contaminated vegetable or water. The eggs of $T$. solium can remain viable for up to 2 months in water, soil or vegetation. Once the eggs are in the intestinal tract, the actions of bile and pancreatic enzymes dissolve their protective coatings. Liberated from their coats, they become embryos (oncospheres), penetrate the intestinal wall and enter the bloodstream. There they are carried to the tissues of the host, where they eventually localize as cysts within the brain, the skeletal muscles, and the eyes and become established as cystic larvae or cysticerci [5].

The former described infection differs from taeniosis. 
Taeniosis in humans results from consumption of improperly cooked meat from swine that are infected by cysticerci (viable larvae) [6]. Taeniosis is usually benign, although it may produce abdominal discomfort and peripheral eosinophilia.

\subsection{Clinical Presentation}

From the moment a cysticercus invades the brain tissue, for example, it is exposed to a hostile environment. When the host's immune system recognizes the parasite as foreign, it usually mounts an appropriate inflammatory reaction to overcome the infection. In many cases, however, such a vigorous response does not occur, and the host reaches a state of immunologic tolerance to the parasite, leaving it almost undisturbed for many years. No clear explanation exists for the individual differences observed in the severity of immunologic response against infection of the central nervous system by cysticerci [6].

Clinical manifestations of neurocysticercosis depend on the number, size, and location of CNS lesions and on the intensity of the host immune response $[7,8]$.

Neurocysticercosis can cause many neurologic symptoms. Late-onset epilepsy is the most common clinical symptom; neurocysticercosis is the leading cause of epilepsy worldwide [9].

Patients with neurocysticercosis may also present with headache associated with intracranial hypertension (another common presentation in disease-endemic countries), focal signs or dementia. Intracranial hypertension is most commonly caused by hydrocephalus related to granular ependymitis, arachnoiditis or ventricular cysts. Other causes include giant cysts or cysticercotic encephalitis.

The course of disease may remain sub acute or chronic for many years, then present with focal signs of a cerebrovascular event secondary to an acute inflammatory response to the parasites. Cysticerci can also invade the spinal cord, the eyes and the subcutaneous and muscular tissues of the body. Muscular pseudo hypertrophy is seen more frequently in patients from Asia who has cysticercosis; it accounts for $0.6 \%$ of cases in China, but is extremely rare in America [6].

\subsection{Diagnosis}

\subsubsection{Pathologic Examination}

Detection of the parasite in a biopsy of a brain or spinal cord lesion is one of the proposed absolute criteria for the diagnosis of neurocysticercosis [10-12].

Grossly, the lesion shows a fibrous capsule, easily demarcate from adjacent brain tissue, when sectioned is cystic; the cyst contents may be grumous and nondescript, but occasionally have recognizable organism-like shapes.

Histologically, cysticerci manifest 4 distinct stages in addition to the racemose form; vesicular, colloidal, granular and calcified $[20,18]$. The vesicular stage consists of vesicles with viable organisms. Each viable organism is composed of a larva containing an invaginated scolex (head) and is surrounded by translucent fluid that is lined by a thin membranous wall $[18,20,23]$. The reactive inflammatory response and associated changes, which are more likely to correlate with clinical symptoms, are usually not elicited with viable cysticerci but instead are found with degenerating cysts $[19,23]$. Depending on the inflammatory process, cysticerci have been documented to survive in the brain for up to several years.

The inflammatory response induced by degenerating cysticerci consists predominantly of lymphocytes and plasma cells, with variable edema, gliosis, fibrosis, and necrosis $[7,9,18]$. The degeneration process begins with the colloidal stage. 7 The vesicle fluid becomes more turbid and the larvae become hyalinized. The inflammatory infiltrate becomes more intense, extending to surrounding structures, and includes lymphocytes, some neutrophils and eosinophils, and the formation of multinucleated histiocytic giant cells $[9,22]$. The reactive inflammatory process can cause arteritis, arachnoiditis, and ependymitis [7]. The granular-nodular stage involves progressive degenerative changes with increased larval decay, vesicle involution, and thickening of the vesicle wall $[7,19]$. The larvae in this stage begin to mineralize with calcium [18]. The degenerative process ends with the nodular-calcified stage. In this stage, cysticerci are replaced by collagen and calcify $[18,19]$. Although this stage is associated with decreased inflammation, foreign body giant cells and gliosis may persist $[7,18]$.

The racemose form of neurocysticercosis most likely represents cysticercal decay with cyst enlargement and hydropic changes [20,22]. Racemose cysticerci usually do not contain scolices and are considered nonviable encysted organisms. The degenerated vesicle wall is characteristically convoluted with external bulbous projections and evokes an inflammatory response [18,22]. The associated reactive process elicited by the racemose form can also cause meningitis [20,22].

\subsubsection{Imaging Studies}

Apart from histologic examination the diagnosis of neurocysticercosis is based on results of neuroimaging studies. Computed tomography is the most commonly used neuroimaging test for the disease and maintains relatively good diagnostic sensitivity when used in disease-endemic areas. Viable cysts are non-enhancing, either in leptomeninges or parenchyma. After several (3 - 30) years, the organism dies and the cyst starts to degenerate and enhance. The cyst wall thickness due to inflamma- 
tion forms a fibrous capsule and the cyst fluid become denser. Eventual cyst shrinkage can take months to years with replacement by a small calcification. Calcifications in the brain parenchyma are the most common finding in computed tomography studies and, in many cases, the only radiologic evidence of the disease. For that reason, computed tomography remains the best screening tool for assessing patients with suspected neurocysticercosis. Figures 1(a) and (b), representing our case CT imaging, show small cystic lesion in parietal and occipital region.

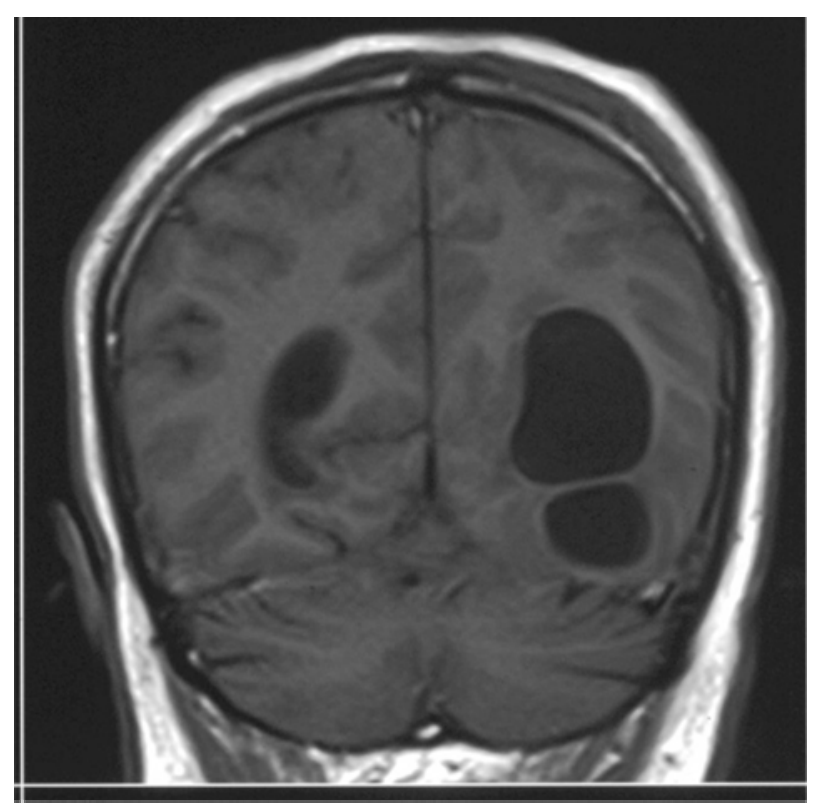

(a)

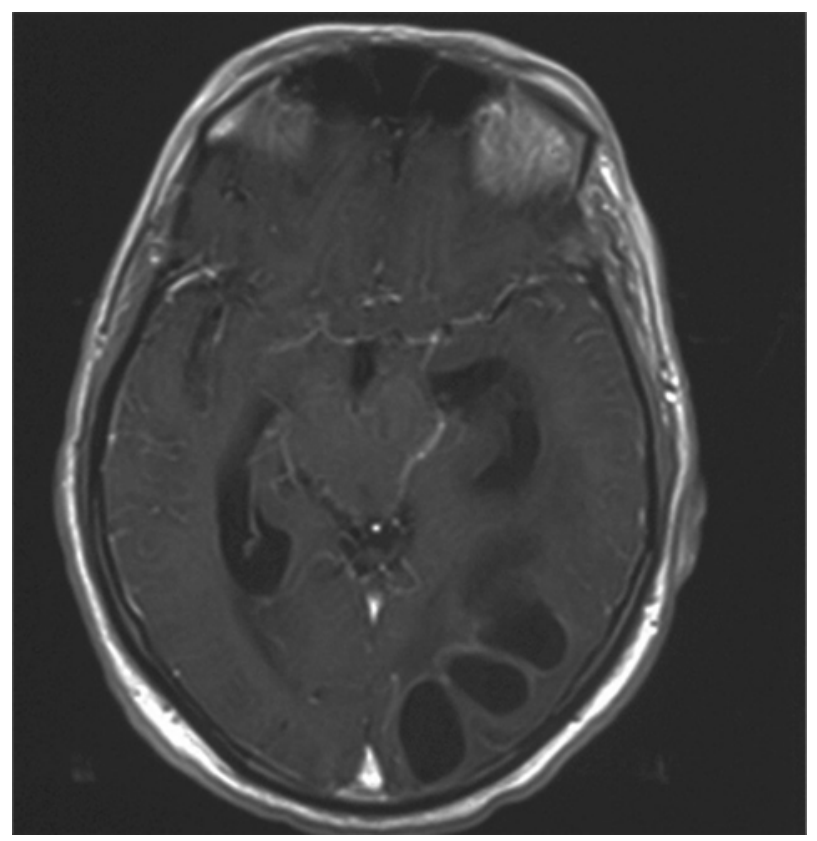

(b)

Figure 1. CT scan imaging of cystic neurocystisercosis lesions.
Small lesions, especially those situated close to bone or within ventricles, may be missed on computed tomography scans. A magnetic resonance imaging scan is therefore often added for increased diagnostic sensitivity and accuracy. Magnetic resonance imaging is also the modality of choice when evaluating patients with intraventricular cysticercosis and when assessing brainstem cysts and small cysts located over the convexity of the cerebral hemispheres. The main shortcoming of magnetic resonance imaging is its failure to detect small calcifications.

The appearance of cysticerci in brain parenchyma on neuroimaging scans depends on their stage of development. Vesicular cysts appear as rounded lesions with signal properties similar to those of cerebrospinal fluid in both $T 1$ - and T2-weighted images. The scolex may be seen within the cyst as a high-intensity nodule, giving the lesion a pathognomonic "hole-with-dot" appearance.

When the parasite begins to degenerate, the lesion becomes heterogeneous, and its appearance varies depending on the degree of degeneration. Cysticercotic encephalitis involves multiple cysts in the brain parenchyma associated with severe, diffuse inflammation.

Cysticercosis may present with a single intraparenchymal brain lesion with nodular or ring enhancement detected with the use of contrast in neuroimaging studies. The differential diagnosis for this type of lesion includes brain tumors, hydatidosis, multiple sclerosis and tuberculomas.

$T$. solium larvae occasionally are deposited in ventricular spaces or in the sub arachnoid space. There, free from surrounding solid tissues, the cyst walls, which develop are often more delicate, and also can grow larger and into more complex shapes. This behavior is described by the term "racemose" cysticercosis.

\subsubsection{Other Findings}

Hematologic abnormalities in patients with neurocysticercosis include mild leukocytosis and eosinophilia in up to $30 \%$ of patients. Stool examination for T. solium eggs is positive in only $5 \%-10 \%$ of patients. In a similar proportion of cases, a T. solium carrier can be found in the patient's close environment. Because analysis of cerebrospinal fluid shows abnormalities in $50 \%$ or less of patients with neurocysticercosis, a normal finding on examination of cerebrospinal fluid does not rule out neurocysticercosis.

Serologic assays, mainly enzyme-linked immunoelectron transfer blot, are also commonly used to detect specific antibodies.

Epidemiologic factors that may lead to a diagnosis of neurocysticercosis include evidence of household contact with $T$. solium, immigration from an area where the disease is endemic and a history of repeated travel to dis- 
ease-endemic regions. An accurate and complete patient history is crucial for neurocysticercosis identification. Patients should be questioned about recent travel. Immigrants are in high risk for bringing this condition with them from less-developed countries. However, with the increase in world travel, persons who would previously not have been exposed to parasitic illnesses such as neurocysticercosis are now susceptible [12] .The use of epidemiologic information together with clinical suspicion and laboratory tests is essential in making the final diagnosis and starting treatment.

However, the combination of epidemiologic, clinical and laboratory information may lead to an accurate diagnosis and avoid an invasive diagnostic procedure.

Neurocysticercosis should be included in the differential diagnosis of neurologic infections in HIV patients in endemic populations. Consideration of the patient's immune status should alert the clinician to potential atypical presentations. Despite the wide endemicity of cysticercosis and HIV infection, less than 30 cases of neurocysticercosis have been reported in HIV infected patients [11].

\subsection{Treatment and Prognosis}

Pharmaceutical treatments for symptoms include analgesics, corticosteroids, and anti seizure medications [17]. Anti parasitic medications are generally effective but there have been rare treatment-related deaths (1\% - 4\%) in cases with hydrocephalus, intracranial hypertension, and massive parasitic loads $[7,24]$.

Placement of a ventriculoperitoneal shunt is indicated in cases of intracranial hypertension before the use of antiparasitic drugs, and surgical removal may be appropriate for large or intraventricular cysts [7,19,24]. Shunt dysfunction rate is also high (30\% - 67\%) [14]. Surgery eliminates the inflammatory nidus, potentially obviating the need for a complication prone shunt. Treatment of intraventricular cysticercosis is a contentious issue. Medical management is less favored because of poor cysticidal CSF penetration, delay in response and, lysis of the cyst leading to ependymitis with resultant acute complications and chronic sequelae [13].

Open surgery was the only available modality previously but now neuroendoscopy has become the treatment of choice $[15,16]$.

The prognosis of is multifactorial and ultimately depends on the host immune response, disease duration, and parasite location, load, size, and stage [7,21,25]. Intraventricular and basal subarachnoid neurocysticercosis have a worse prognosis due to obstruction of cerebrospinal fluid outflow leading to hydrocephalus and increased intracranial pressure $[19,23]$. In addition, the racemose form of neurocysticercosis has a high mortality rate [23].

\section{Discussion}

In our case, the inflamed fibrous wall encircles the contents and as are not wholly necrotic and degenerated, are readily recognizable as non-human tissue. The outer wall of these invertebrate multi cellular parasites is an acellular exoskeleton of chitin. Under that wall, there is a layer of small round cells; deep to, that is the gut of the organism with atypical glandular epithelium thrown into many folds (Figures 2(a) and (b)).

This appearance is typical for cysticerci but is not diagnostic for $T$. solium as compared to other types of metazoan parasites. Correct species identification depends on characterization of the scolices of the larva, but these are rarely completely or even partially visualized in tissue sections, so that precise identification may require other studies like DNA analysis and immunoassays. As treatment and prognosis are independent of species identification, this is not generally done. Other parasites which may be encountered include Sparganosis, Tri-

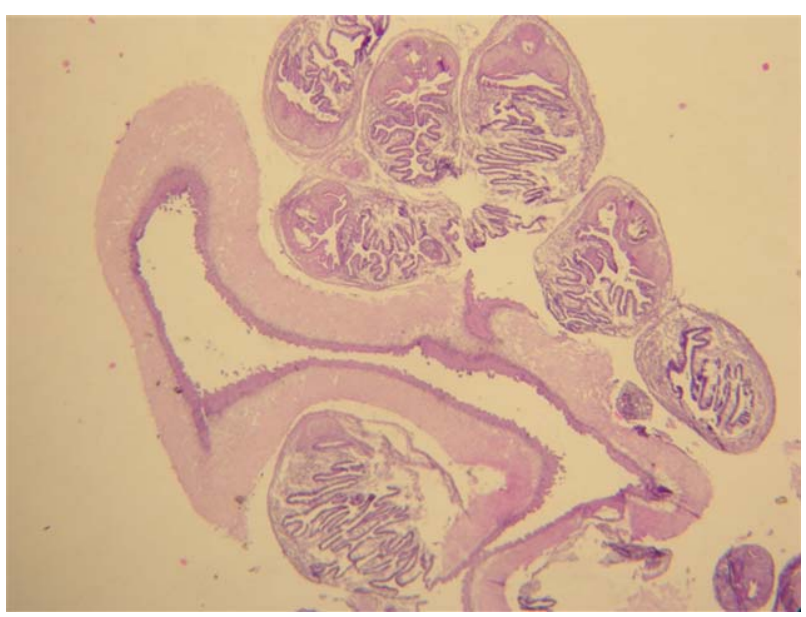

(a)

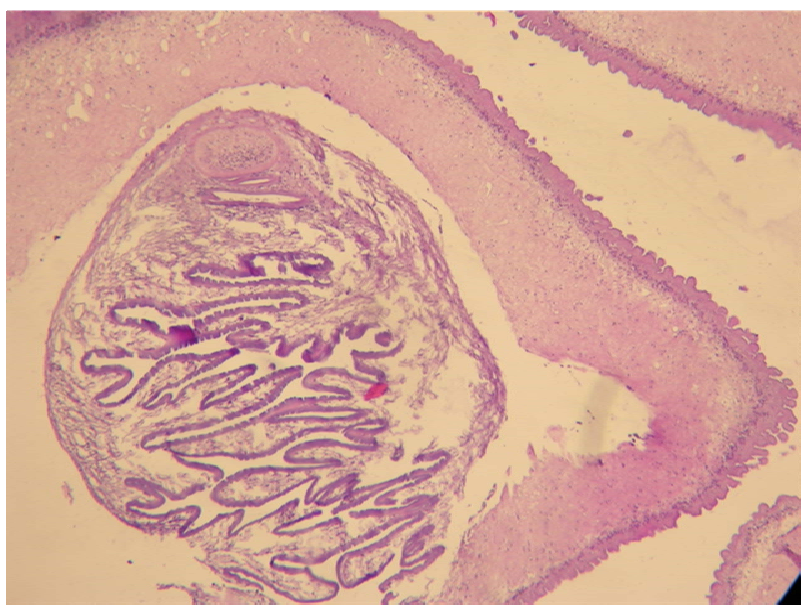

(b)

Figure 2. Low and high power view of the parasites (H \& E staining). 
chionosis, and Echinococcus cysts.

\section{REFERENCES}

[1] M. Gemmell, Z. Matyas, Z. Pawlowsky and E. J. L. Soulsby, "Guidelines for Surveillance and Control of Taeniasis/Cysticercosis, Vol. 83,” World Health Organization, Switzerland, 1983, p. 49.

[2] G. Roman, J. Sotelo, O. Del Brutto, et al., “A Proposal to Declare Neurocysticercosis an International Reportable Disease," Bulletin of the World Health Organization, Vol. 78, No. 3, 2000, pp. 399-406

[3] K. Hutcheson III, M. Kalafian, I. Taylor, J. Sagel and B. Clyburn, "Neurocysticercosis," Southern Medical Journal, Vol. 7, 2000, pp. 666-668.

[4] M. T. Wallin and J. F. Kurtzke, "Neurocysticercosis in the United States: Review of an Important Emerging Infection,” Neurology, Vol. 63, No. 9, 2004, pp. 1559-1564. http://dx.doi.org/10.1212/01.WNL.0000142979.98182.FF

[5] O. H. Del Brutto, J. Sotelo, "Neurocysticercosis: An Update,” Clinical Infectious Disease, Vol. 10, No. 6, 1988, pp. 1075-1087.

[6] J. G. Burneo, I. Plener and H. H. Garcia, "Neurocysticercosis in a Patient in Canada," CMAJ, Vol. 180, No. 6, 2009, pp. 639-642.

[7] H. H. Garcia and O. H. Del Brutto "Neurocysticercosis: Updated Concepts about an Old Disease," The Lancet Neurology, Vol. 4, No. 10, 2005, pp. 653-661. http://dx.doi.org/10.1016/S1474-4422(05)70194-0

[8] J. A. Serpa, L. S. Yancey and A. C. White Jr., "Advances in the Diagnosis and Management of Neurocysticercosis," Expert Review of Anti-infective Therapy, Vol. 4, No. 6, 2006, pp. 1051-1061.

http://dx.doi.org/10.1586/14787210.4.6.1051

[9] H. H. Garcia, R. Gilman, M. Martinez, et al., "Cysticercosis as a Major Cause of Epilepsy in Peru. The Cysticercosis Working Group in Peru (CWG)," Lancet, Vol. 341, No. 8839, 1993, pp. 197-200. http://dx.doi.org/10.1016/0140-6736(93)90064-N

[10] O. H. Del Brutto, V. Rajshekhar, A. C. White Jr., et al., "Proposed Diagnostic Criteria for Neurocysticercosis," Neurology, Vol. 57, No. 2, 2001, pp. 177-183. http://dx.doi.org/10.1212/WNL.57.2.177

[11] J. A. Serpa, A. Moran, J. C. Goodman, T. P. Giordano, and A. C. White Jr, "Neurocysticercosis in the HIV Era: A Case Report and Review of the Literature," American Journal of Tropical Medicine and Hygiene, Vol. 77, No. 1, 2007, pp. 113-117.

[12] S. Remy, MSN, RN, CEN and Vineland, “A 44-Year-Old Man with Neurocysticercosis,” Journal of Emergency Nursing, Vol. 35, No. 4, 2009, pp. 348-349. http://dx.doi.org/10.1016/j.jen.2008.04.024

[13] S. A. Cudlip, P. R. Wilkins and H. T. Marsh, "Endoscopic Removal of a Third Ventricular Cysticercal Cyst," British Journal of Neurosurgery, Vol. 12, No. 5, 1998, pp.
452-454. http://dx.doi.org/10.1080/02688699844691

[14] R. Kelley, D. H. Duong and G. E. Locke, “Characteristics of Ventricular Shunt Malfunctions among Patients with Neurocysticercosis,” Neurosurgery, Vol. 50, No. 4, 2002, pp. 757-762. http://dx.doi.org/10.1097/00006123-200204000-00014

[15] M. Bergsneider, L. T. Holly, J. H. Lee, W. A. King and J. G. Frazee, "Endoscopic Management of Cysticercal cysts within the Lateral and Third Ventricles," Journal of Neurosurgery, Vol. 92, No. 1, 2000, pp. 14-23. http://dx.doi.org/10.3171/jns.2000.92.1.0014

[16] V. Kalra, D. Mishra, A.Suri, R. Seth and A. Garg, "Intraventricular Neurocysticercosis," The Indian Journal of Pediatrics, Vol. 76, No. 4, 2009, pp. 420-423.

[17] H. H. García, C. A. Evans, T. E. Nash, O. M. Takayanagui, A. C. White, D. Botero, V. Rajshekhar, V. C.Tsang, et al., "Current Consensus Guidelines for Treatment of Neurocysticercosis,” Clinical Microbiology Reviews, Vol. 15, No. 4, 2002, pp. 747-756.

http://dx.doi.org/10.1128/CMR.15.4.747-756.2002

[18] A. Escobar and K. M. Weidenheim, "The Pathology of Neurocysticercosis,” In: G. Singh and S. Prabhakar, Eds., Taenia solium Cysticercosis: From Basic to Clinical Science, United Kingdom CABI Publishing, Wallingford, 2002, pp. 289-305. http://dx.doi.org/10.1079/9780851996288.0289

[19] A. C. White Jr., "Neurocysticercosis: Updates on Epidemiology, Pathogenesis, Diagnosis, and Management," Annual Review of Medicine, Vol. 51, 2000, pp. 187-206. http://dx.doi.org/10.1146/annurev.med.51.1.187

[20] A. Carpio, “Neurocysticercosis: An Update," The Lancet Infectious Diseases, Vol. 2, No. 12, 2002, pp. 751-762. http://dx.doi.org/10.1016/S1473-3099(02)00454-1

[21] M. P. Earnest, L. B. Reller, C. M. Filley and A. J. Grek, "Neurocysticercosis in the United States: 35 Cases and a Review,” Reviews of Infectious Diseases, Vol. 9, No. 5, 1987, pp. 961-979. http://dx.doi.org/10.1093/clinids/9.5.961

[22] J. E. Pittella, “Neurocysticercosis,” Brain Pathology, Vol. 7, No. 1, 1997, pp. 681-693. http://dx.doi.org/10.1111/j.1750-3639.1997.tb01083.x

[23] H. H. García, A. E. Gonzalez, C. A. Evans and R. H. Gilman, "Cysticercosis Working Group in Peru. Taenia solium Cysticercosis,” Lancet, Vol. 362, No. 9383, 2003, pp. 547-556. http://dx.doi.org/10.1016/S0140-6736(03)14117-7

[24] C. M. DeGiorgio, M. T. Medina, R. Durón , C. Zee and S. P. Escueta, "Neurocysticercosis," Epilepsy Currents, Vol. 4, No. 3, 2004, pp. 107-111. http://dx.doi.org/10.1111/j.1535-7597.2004.43008.x

[25] D. Scharf, "Neurocysticercosis: Two Hundred ThirtyEight Cases from a California Hospital," Archives of Neurology, Vol. 45, No. 7, 1988, pp. 777-780. http://dx.doi.org/10.1001/archneur.1988.00520310087022 